\title{
A slow bow shock ahead of the heliosphere
}

\author{
B. Zieger, ${ }^{1}$ M. Opher, ${ }^{1}$ N. A. Schwadron,,${ }^{1,2,3}$ D. J. McComas, ${ }^{3,4}$ and G. Tóth ${ }^{5}$ \\ Received 16 April 2013; revised 17 May 2013; accepted 16 May 2013; published 21 June 2013.
}

[1] Current estimates of plasma parameters in the local interstellar medium indicate that the speed of the interstellar wind, i.e., the relative speed of the local interstellar cloud with respect to the Sun, is most likely less than both the fast magnetosonic speed (subfast) and the Alfvén speed (sub-Alfvénic) but greater than the slow magnetosonic speed (superslow). In this peculiar parameter regime, MHD theory postulates a slow magnetosonic shock ahead of the heliosphere, provided that the angle between the interstellar magnetic field and the interstellar plasma flow velocity is quite small (e.g., $15^{\circ}$ to $30^{\circ}$ ). In this likely scenario, our multifluid MHD model of the heliospheric interface self-consistently produces a spatially confined quasi-parallel slow bow shock. Voyager 1 is heading toward the slow bow shock, while Voyager 2 is not, which means that the two spacecraft are expected to encounter different interstellar plasma populations beyond the heliopause. The slow bow shock also affects the density and spatial extent of the neutral hydrogen wall. Citation: Zieger, B., M. Opher, N. A. Schwadron, D. J. McComas, and G. Tóth (2013), A slow bow shock ahead of the heliosphere, Geophys. Res. Lett., 40, 2923-2928, doi:10.1002/grl.50576.

\section{Introduction}

[2] The plasma parameters in the local interstellar medium (LISM) are becoming more constrained due to direct observations (for a recent review, see Frisch et al. [2011]). Nevertheless, there is no general consensus regarding the strength and direction of the interstellar magnetic field $\left(\mathbf{B}_{\text {ISM }}\right)$, since competing models predict significantly different $B_{\text {ISM }}$ values. Interstellar neutral He measurements onboard Ulysses [Witte, 2004] provided an estimate of $26.3 \mathrm{~km} / \mathrm{s}$ for the bulk speed of the pristine interstellar wind. The latest results from the Interstellar Boundary Explorer (IBEX) indicate an even lower value of $23.2 \mathrm{~km} / \mathrm{s}$ [McComas et al., 2012].

[3] The termination shock crossings of the two Voyager spacecraft were used to constrain the pristine interstellar magnetic field in multifluid MHD models of the heliospheric interface, suggesting a significantly stronger $\mathbf{B}_{\mathrm{ISM}}$

\footnotetext{
${ }^{1}$ Center for Space Physics, Boston University, Boston, Massachusetts, USA.

${ }^{2}$ Institute for the Study of Earth, Oceans, and Space, University of New Hampshire, Durham, New Hampshire, USA.

${ }^{3}$ Southwest Research Institute, San Antonio, Texas, USA.

${ }^{4}$ Physics and Astronomy Department, University of Texas, San Antonio, Texas, USA.

${ }^{5}$ Department of Atmospheric, Oceanic, and Space Sciences, University of Michigan, Ann Arbor, Michigan, USA.

Corresponding author: B. Zieger, Boston University, 725 Commonwealth Avenue, Boston, MA 02215, USA. (berci@bu.edu)

(C)2013. American Geophysical Union. All Rights Reserved. 0094-8276/13/10.1002/grl.50576
}

(3.7-5.5 $\mu \mathrm{G})$ than previous estimates $(1.8-2.5 \mu \mathrm{G})$ and a relatively small angle $\left(\alpha_{B v}=15^{\circ}-30^{\circ}\right)$ between $\mathbf{B}_{\text {ISM }}$ and the flow velocity of the interstellar wind ( $\left.\mathbf{v}_{\mathrm{ISM}}\right)$ [Opher et al., 2009]. Similar strong $B_{\mathrm{ISM}}$ intensities and small $\alpha_{B v}$ angles were obtained in kinetic-gasdynamic models as well [Izmodenov et al., 2009].

[4] There are constraints based on models of the IBEX ribbon. However, these models assume particular physical mechanisms for the ribbon. Works such as Heerikhuisen and Pogorelov [2011] argue that in order to get agreement with the observed shape of the ribbon, $B_{\text {ISM }}$ must be not more than $3 \mu \mathrm{G}$. However, they strongly rely on the Heerikhuisen et al. [2010] mechanism. A number of ribbon models find that $\mathbf{B}_{\text {ISM }}$ should point in the direction of the ribbon arc center, which gives $\alpha_{B v}=45^{\circ}$ [McComas et al., 2009; Schwadron et al., 2009; Heerikhuisen et al., 2010; Schwadron and McComas, 2013]. However, other ribbon models, e.g., the one by Chalov et al. [2010], obtain that $B_{\text {ISM }}$ should be strong $(4.4 \mu \mathrm{G})$ and $\alpha_{B v}$ should be small $\left(20^{\circ}\right)$. Since a comprehensive model for the physics of the ribbon source region is still missing, the nature of LISM-heliosphere interaction cannot be nailed down yet.

[5] As Pogorelov et al. [2006] demonstrated in a numerical study, there are two possible solutions for the LISMheliosphere interaction, the superfast interaction, which results in a fast magnetosonic bow shock, and the subfast interaction, which can result in a slow magnetosonic bow shock, hereafter referred to as slow bow shock or SBS. The same total pressure is obtained at the nose of the heliosphere for a weak $B_{\text {ISM }}$ with large $\alpha_{B v}\left(1.5 \mu \mathrm{G}\right.$ and $\left.45^{\circ}\right)$ in one case and for a strong $B_{\mathrm{ISM}}$ with small $\alpha_{B v}\left(4 \mu \mathrm{G}\right.$ and $\left.15^{\circ}\right)$ in the other case. In the present study, we adopt a strong $B_{\text {ISM }}$ of $4.37 \mu \mathrm{G}$ and a small $\alpha_{B v}$ of $15.9^{\circ}$ in order to ensure agreement with the Voyager observations of heliospheric asymmetries. This corresponds to the subfast interaction type, i.e., the slow bow shock scenario, as demonstrated in section 3 .

[6] It has been shown recently that the interstellar wind is most certainly sub-Alfvénic, and consequently, no regular fast magnetosonic bow shock can form upstream of the heliosphere [McComas et al., 2012]. Fast bow shocks are general features of magnetized planets in the solar system, which are typically strong shocks (with high compression ratio) due to the high fast magnetosonic Mach number in the solar wind. However, the unique plasma environment of the LISM with a steady sub-Alfvénic and superslow plasma flow is favorable for the formation of a slow bow shock, if $\alpha_{B v}$ is sufficiently small. This likely scenario was not considered by McComas et al. [2012], and therefore their final conclusion of the lack of a bow shock referred exclusively to a classic fast magnetosonic bow shock.

[7] In this letter, the likely existence of a quasi-parallel SBS ahead of the heliosphere is demonstrated both on theoretical basis and by means of a 3-D steady state multifluid 
MHD simulation. Finally, several interesting implications of a SBS are discussed.

\section{Theoretical Considerations}

[8] Here we adopt the latest consensus values of LISM plasma parameters [Frisch et al., 2011] and vary the strength and direction of $\mathbf{B}_{\mathrm{ISM}}$ in order to determine the nature of LISM-heliosphere interaction as a function of $\mathbf{B}_{\mathrm{ISM}}$. More specifically, we assume $n_{p}=0.07 \mathrm{~cm}^{-3}, n_{H}=0.19 \mathrm{~cm}^{-3}$, $T=6300 \mathrm{~K}$, and $v_{\mathrm{ISM}}=23.2$ to $26.4 \mathrm{~km} \mathrm{~s}^{-1}$, where $n_{p}$ and $n_{H}$ are proton and neutral hydrogen number densities, and $T$ is the equilibrium temperature assumed for all components of the LISM. The phase velocities of fast/slow magnetosonic waves for three different values of $B_{\text {ISM }}(2,3$, and $4 \mu \mathrm{G})$ are plotted in the Friedrichs diagrams of Figure 1 with blue/red dash-dotted, dashed, and solid lines, respectively. This is a polar diagram in velocity space where the radial distance is speed in units of $\mathrm{km} \mathrm{s}^{-1}$ and the azimuthal angle shows the direction of the velocity vector with respect to the ambient magnetic field $\left(\mathbf{B}_{\mathrm{ISM}}\right)$. The color shaded area represents the velocity space of $\mathbf{v}_{\text {ISM }}$ ranging from 23.2 to $26.3 \mathrm{~km} \mathrm{~s}^{-1}$ in magnitude and from $15^{\circ}$ to $50^{\circ}$ in $\alpha_{B v}$. While fast magnetosonic waves can propagate in all directions at comparable speeds, slow magnetosonic waves cannot propagate perpendicular to the magnetic field. If the shaded area of $\mathbf{v}_{\text {ISM }}$ falls inside or outside a blue/red curve, it means that fast/slow magnetosonic waves can propagate faster or slower in those directions than the speed of the interstellar wind. From this diagram, one can conclude that for $B_{\mathrm{ISM}}$ greater than $3.2 \mu \mathrm{G}$, the interstellar wind is subfast and superslow, resulting in a slow bow shock. On the other hand, $B_{\mathrm{ISM}}$ must be less than $2.5 \mu \mathrm{G}$ to remain superfast, producing a fast bow shock. This result is consistent with the findings of McComas et al. [2012].

[9] Although slow magnetosonic waves can propagate in any direction except perpendicular to the ambient magnetic field, slow-mode wave packets propagate at the group velocity only within a narrow angle to the ambient magnetic field. Therefore, the slow magnetosonic information about the heliopause is transmitted upstream in the interstellar wind only along magnetic filed lines. As a consequence, the center of the SBS must be displaced from the axis (nose direction) of the heliopause toward the direction of $\mathbf{B}_{\mathrm{ISM}}$.

[10] In a low- $\beta$ plasma, MHD theory predicts a slow shock in the sub-Alfvénic and superslow flow regime, where the Alfvén Mach number $\left(M_{a}\right)$ is less than 1 and the sonic Mach number $\left(M_{s}\right)$ is greater than 1 , which is often referred to as the first elliptic regime [Kogan, 1959]. The compression ratio $(q)$ of a first elliptic slow shock is always greater than 1, which implies that the SBS cannot degenerate into a slow-mode wave characteristic extending to infinity at the weak shock limit $(q=1)$. This means that the SBS will be spatially restricted, not extending to infinity along the asymptotic cone as regular fast bow shocks do.

[11] The existence of an axisymmetric SBS ahead of the heliosphere in case of a strong $\mathbf{B}_{\mathrm{ISM}}(4-5 \mu \mathrm{G})$ parallel to $\mathbf{v}_{\text {ISM }}$ was first suggested by Florinski et al. [2004], based on a 2-D multifluid MHD simulation. This study was motivated by the theoretical work of Cox and Helenius [2003], who predicted a strong $\mathbf{B}_{\text {ISM }}$ of 5 to $7 \mu \mathrm{G}$ pointing toward the $\mathrm{G}$ cloud on the basis of their fluxtube model of the local fluff in

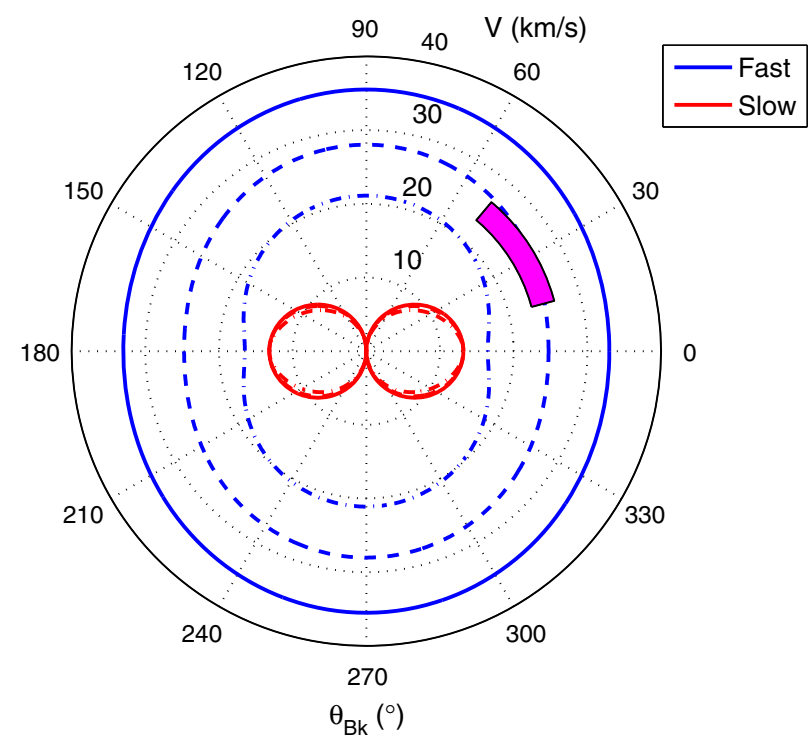

Figure 1. Friedrichs diagram of phase velocities of fast and slow magnetosonic waves in the pristine LISM. The radial coordinate corresponds to the velocity magnitude, with the dotted circles marking the 10,20 , and $30 \mathrm{~km} \mathrm{~s}^{-1}$ values. The polar angle corresponds to the velocity direction relative to $\mathbf{B}_{\text {ISM }}$. The blue and red lines correspond to fast and slow magnetosonic waves in velocity space. Dash-dotted, dashed, and solid lines represent magnetosonic waves for $B_{\mathrm{ISM}}$ of 2,3 , and $4 \mu \mathrm{G}$, respectively. The colored area shows the likely velocity range of the interstellar wind in velocity space, ranging from 23.2 to $26.3 \mathrm{~km} \mathrm{~s}^{-1}$ in speed and making an angle between 15 and $50^{\circ}$ with respect to $\mathbf{B}_{\mathrm{ISM}}$.

the Local Bubble. Pogorelov et al. [2006] confirmed that a SBS may exist in the subfast interaction regime with strong $B_{\text {ISM }}$ and small $\alpha_{B v}$ angle. However, the discontinuity of the SBS was not resolved in these early simulations because of insufficient grid resolution. In the following section, we present numerical simulation results for a plausible SBS scenario with $B_{\mathrm{ISM}}$ of $4.37 \mu \mathrm{G}$ and $\alpha_{B v}$ of $15.9^{\circ}$, which provides the best fit to observed heliospheric asymmetries according to multifluid MHD simulations by Opher et al. [2009].

\section{Numerical Simulation Results}

[12] We use our most recent multifluid, multi-ion MHD model of the heliospheric interface [Prested et al., 2012] built on the BATS-R-US code [Tóth et al., 2012] to simulate the theoretically predicted SBS. The model parameters for the LISM are $n_{p}=0.06 \mathrm{~cm}^{-3}, n_{H}=0.18 \mathrm{~cm}^{-3}, T=$ $6519 \mathrm{~K}$, and $v_{\mathrm{ISM}}=26.4 \mathrm{~km} \mathrm{~s}^{-1}$ pointing toward $(\lambda, \beta)=$ $\left(75.4^{\circ},-5.2^{\circ}\right)$ in $\mathrm{J} 2000$ ecliptic coordinates, which are not significantly different from the consensus values listed in the previous section. Only the strength $(4.37 \mu \mathrm{G})$ and direction of $\mathbf{B}_{\text {ISM }}$ is different from those used in the superfast interaction models. The $\mathbf{B}_{\text {ISM }}$ direction is defined by $\alpha_{B v}=15.9^{\circ}$ and $\beta_{B v}=51.5^{\circ}$, where $\beta_{B v}$ is the angle between the $\mathbf{B}_{\text {ISM }}-\mathbf{v}_{\text {ISM }}$ plane and the solar heliographic equator. In the coordinate system of our model, the $Z$ axis is along the rotation axis of the Sun, the $X$ axis is selected so that the $X$ - $Z$ plane contains the direction of the interstellar neutral He flow, and the $Y$ axis completes the right-handed coordinate system. The size of 
a

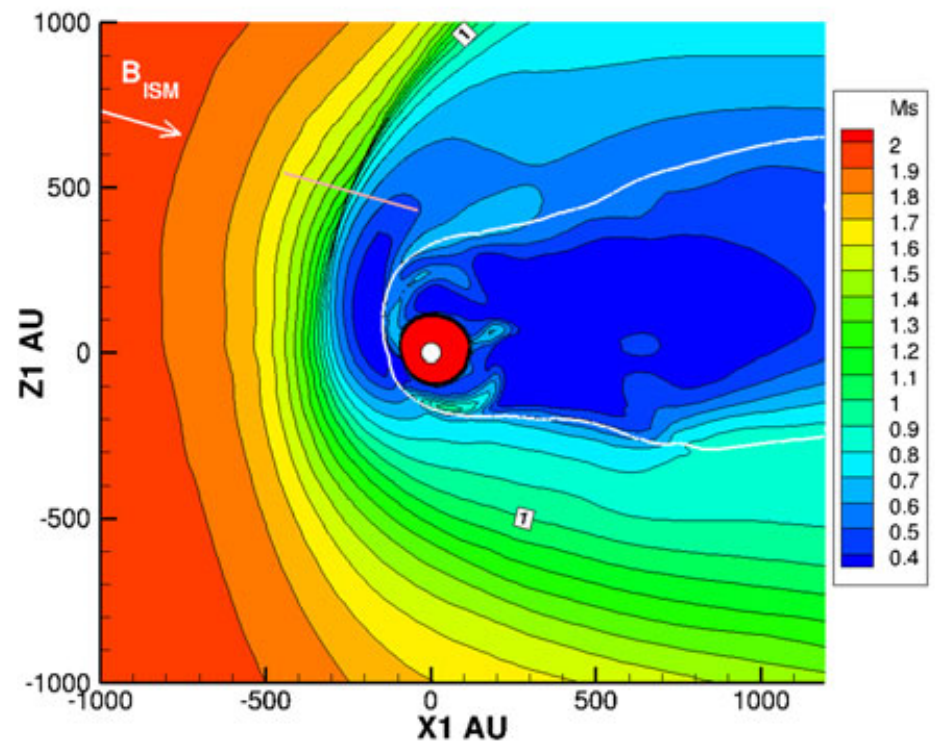

b

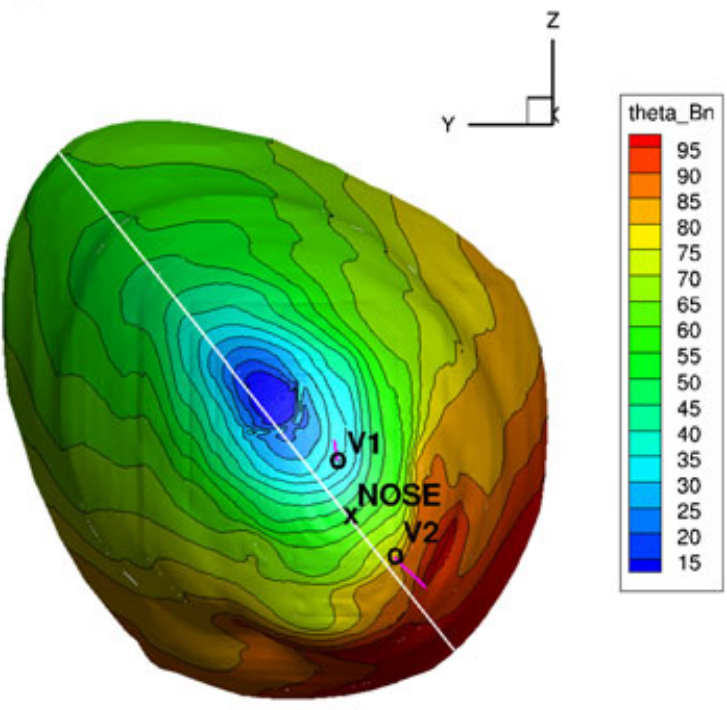

Figure 2. (a) Contour plot of the sonic Mach number in the $\mathbf{B}_{\mathrm{ISM}}-\mathbf{v}_{\mathrm{ISM}}$ plane, where the $X 1$ axis points in the direction of $\mathbf{v}_{\text {ISM }}$ and the $Z 1$ axis is perpendicular to both $\mathbf{v}_{\text {ISM }}$ and the normal to the $\mathbf{B}_{\text {ISM }}-\mathbf{v}_{\text {ISM }}$ plane. The converging contour lines indicate the location of the SBS. The contour line of $M_{s}=1$ is labeled with number 1 . The heliopause is plotted with a white line. The pink line is the trajectory of the quasi-parallel SBS crossing plotted in Figure 3 . The direction of the pristine $\mathbf{B}_{\text {ISM }}$ is shown by a white arrow. (b) A 3-D view of the slow magnetosonic surface with the prospective crossing locations of V1 and V2. The angle between $\mathbf{B}_{\text {ISM }}$ and the normal to this isosurface $\left(\theta_{B n}\right)$ is plotted as contour lines on the surface. The SBS is restricted to the quasi-parallel region of bluish colors northwest of the nose. The white line indicates the intersection of the isosurface with the $\mathbf{B}_{\text {ISM }}-\mathbf{v}_{\text {ISM }}$ plane.

the simulation box is $3000 \mathrm{AU}$ in all three dimensions with the Sun in the center. The inner boundary conditions for the solar wind are set at a radial distance of $30 \mathrm{AU}$. Geometric mesh refinement was applied to resolve the regions of interest, resulting in a smallest cell size of $0.73 \mathrm{AU}$ around the quasi-parallel SBS.

[13] In Figure 2a, we plotted contour lines of $M_{s}$ in the $\mathbf{B}_{\text {ISM-V }}$ ISM plane, where the $X 1$ axis is along $\mathbf{v}_{\text {ISM }}$, and the $Z 1$ axis is perpendicular to both $\mathbf{v}_{\text {ISM }}$ and the normal to the $\mathbf{B}_{\text {ISM }}-\mathbf{v}_{\text {ISM }}$ plane. $M_{S}$ clearly shows a large gradient across the isosurface of $M_{S}=1$ (labeled with 1) in the quasi-parallel region, where $\theta_{B n}$, i.e., the angle between the interstellar magnetic field and the normal to the isosurface, is small. This is the region where slow magnetosonic waves are steepened into a SBS. On the other hand, the transition of $M_{S}$ across the isosurface is smooth in the quasi-perpendicular region (lower part of Figure 2a), as expected from MHD theory. Since slow magnetosonic information of the heliopause can propagate upstream in the interstellar wind only along magnetic field lines (as discussed in section 2), the SBS is strongly offset from the nose direction.

[14] A 3-D view of the slow magnetosonic surface (SMS) in the interstellar wind is illustrated in Figure 2b. The SMS is the surface in the decelerating interstellar wind where the flow speed is equal to the upstream component of the slow magnetosonic group velocity, so that no slow-mode information can propagate any further upstream of this surface. In other words, the SMS is defined as the isosurface where the slow magnetosonic Mach number $M_{\text {slow }}=v_{\text {ISM }} /\left|c_{S} \cos \left(\theta_{B v}\right)\right|$ is equal to 1 . Here $\theta_{B v}$ is the angle between the local magnetic field vector and the flow velocity. A SMS would exist in any decelerating or accelerating MHD wind, and it is not necessarily a shock or discontinuity. It is a physical surface which separates the flow region where points are casually connected via slow magnetosonic waves from the region where they are not [Bogovalov, 1994]. However, in case of an obstacle, like the heliosphere in our case, a part of the SMS can steepen into a slow magnetosonic bow shock (SBS). In the present simulation, the SBS is spatially restricted to the quasi-parallel region of the SMS (bluish region in Figure 2b) northwestward from the nose, being more or less symmetric to the $\mathbf{B}_{\text {ISM }}-\mathbf{v}_{\text {ISM }}$ plane. Thus, the SBS is strongly asymmetric with respect to the axis of the heliosphere.

[15] Interestingly, the prospective crossing of Voyager 1 through the SMS is located in a quasi-parallel region with $\theta_{B n}=35^{\circ}$, while the prospective crossing of Voyager 2 through the same surface is located in a quasi-perpendicular region with $\theta_{B n}=80^{\circ}$ (see Figure $2 \mathrm{~b}$ ). In other words, Voyager 1 (V1) could be heading toward a SBS, while Voyager 2 (V2) is likely not.

[16] The quasi-parallel SBS crossing indicated in Figure 2a (pink line) is presented in the six panels of Figure 3. The shock normal obtained for this crossing is $[-0.8930,0.3072,0.3289]$, which gives a $\theta_{B n}$ of $9^{\circ}$. Distance is measured from the SBS (marked with a vertical black line) in the downstream direction. Due to upstream propagating fast magnetosonic waves, the flow begins to decelerate upstream of the SBS. The interstellar wind then changes abruptly from superslow $\left(M_{s}>1\right)$ to subslow $\left(M_{s}<1\right)$ across the shock while remaining sub-Alfvénic on both sides (Figure 3a).

[17] The density jump (Figure 3c) is in agreement with the theoretical prediction (dashed line). The theoretical 

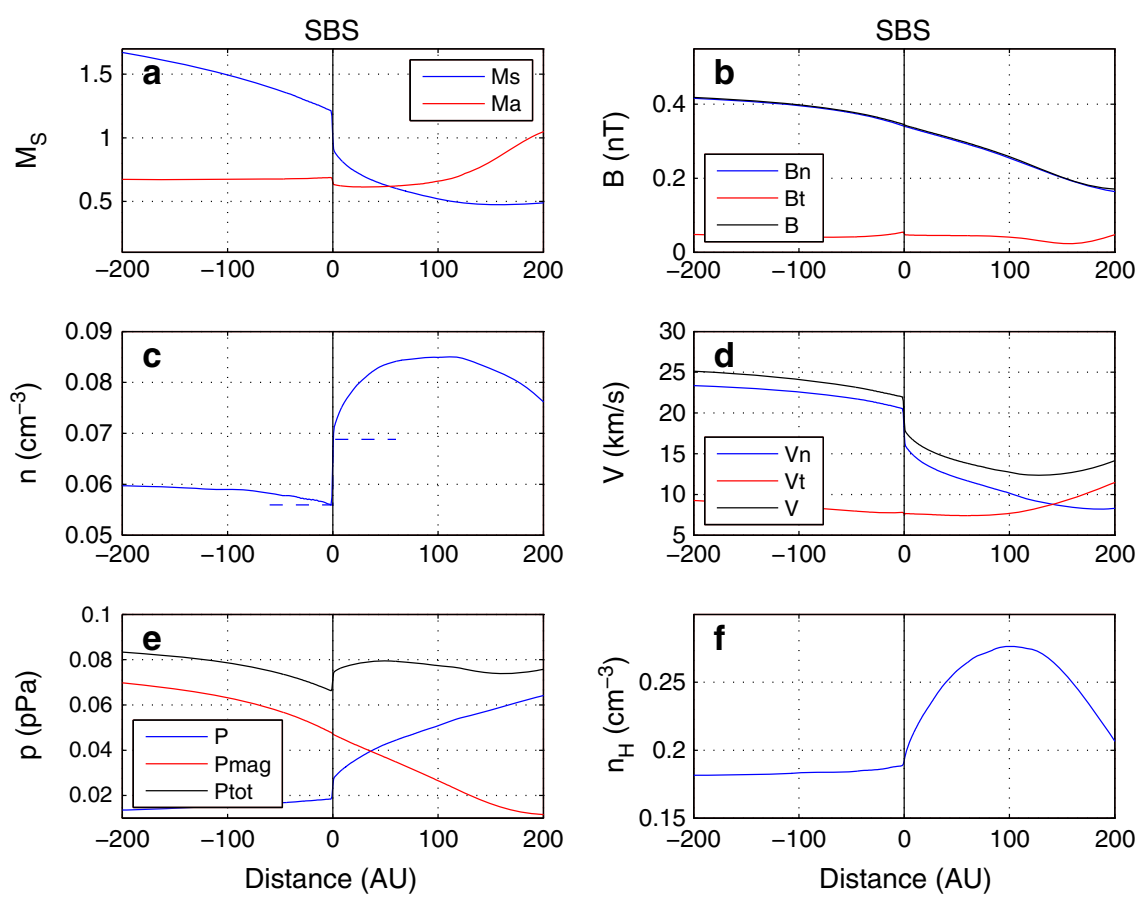

Figure 3. Quasi-parallel SBS crossing along the pink trajectory plotted in Figure 2a. Distance is measured from the SBS (zero point in the figure) in the downstream direction. (a) The sonic and Alfvén Mach numbers, (b) magnetic field components, (c) ion number density, (d) plasma flow velocity components, thermal plasma (blue), magnetic (red), and total (black) (e) pressures, and (f) neutral hydrogen number density (f). The blue dashed line in Figure $3 \mathrm{c}$ is the theoretically predicted density jump across the SBS.

compression ratio of $q=1.23$ was obtained from the general shock adiabatic equation, where the upstream velocity had been transformed into the de Hoffmann-Teller (H-T) frame. Although the shock is relatively weak, it is still strong enough to be theoretically admissible. The upstream Mach numbers in the H-T frame are $M_{s}=1.15$ and $M_{a}=0.65$, which fall apparently in the first elliptic regime where a slow shock solution exists. Thus, we conclude that the simulated discontinuity is indeed a slow shock.

[18] The normal component of the magnetic field does not jump across the SBS as expected from the RankineHugoniot conditions (Figure 3b), while the tangential component shows a slight increase right ahead of the shock before jumping from a higher upstream value to a lower downstream value. The latter is typical of slow shocks, implying a rotation of the magnetic field toward the shock normal. Since the shock is almost parallel $\left(\theta_{B n}=9^{\circ}\right)$, the rotation of the field is very small. Another typical feature of a SBS is the gradual decrease of the magnetic field strength in the downstream direction (see black line in Figure 3b), which is not the case in a fast bow shock scenario. At the heliopause downstream of the SBS, $B_{\text {ISM }}$ drops to less than half of the pristine value, making the plasma $\beta$ greater than 1 .

[19] The normal component of the velocity jumps from superslow to subslow across the shock but remains subAlfvénic, as required for a slow shock (Figure 3d). The thermal plasma pressure shows the expected jump right at the SBS, indicating kinetic energy dissipation through the heating of the thermal ion population. Further downstream, the thermal pressure $(p)$ gradually increases, while the magnetic pressure $\left(p_{\text {mag }}\right)$ gradually decreases, keeping the total pressure $\left(p_{\text {tot }}\right)$ constant across the outer heliosheath (Figure $3 \mathrm{e}$ ).
Eventually, the plasma $\beta$ surpasses 1, and the plasma becomes thermally dominated closer to the heliopause.

[20] Figure $3 \mathrm{f}$ shows the profile of the neutral hydrogen wall in neutral hydrogen number density. The SBS clearly limits the upstream extension of the hydrogen wall, although no discontinuity is observed in the neutral fluid. The latter is not unexpected, since the heliopause is not an obstacle for the neutrals. The hydrogen wall is restricted to the region of the outer heliosheath between the SBS and the heliopause because this is the region where significant velocity difference occurs between the ionized and neutral components of the interstellar wind. Since ions and neutrals communicate through charge exchange, any velocity difference either in bulk velocity or thermal velocity would result in an enhanced charge exchange rate that produces the secondary neutrals of the hydrogen wall. In the lack of a SBS, the hydrogen wall is expected to be lower and thicker because the deceleration of the plasma is gradual and smooth in this case.

[21] Finally, we want to demonstrate in Figure 4 the effect of the SBS on the LISM plasma parameters beyond the heliopause. Since V1 is heading toward the SBS and V2 is not, the two spacecraft will sample different plasma populations in the LISM. V1 will enter a compressed (Figure 4a) and heated (Figure 4c) thermally dominated (compare solid and dashed red lines in Figure $4 \mathrm{~b}$ ) outer heliosheath, where the plasma $\beta$ is greater than 1 (red line in Figure $4 d$ ). Unlike V1, V2 will encounter a magnetically dominated (compare solid and dashed blue lines in Figure 4b) low- $\beta$ (blue line in Figure $4 d$ ) LISM population, where the plasma $\beta$ is less than 1 , like in the pristine LISM $(\beta=0.14)$. The most dramatic difference in LISM parameters between V1 and V2 right outside the heliopause is the 1 order of magnitude 

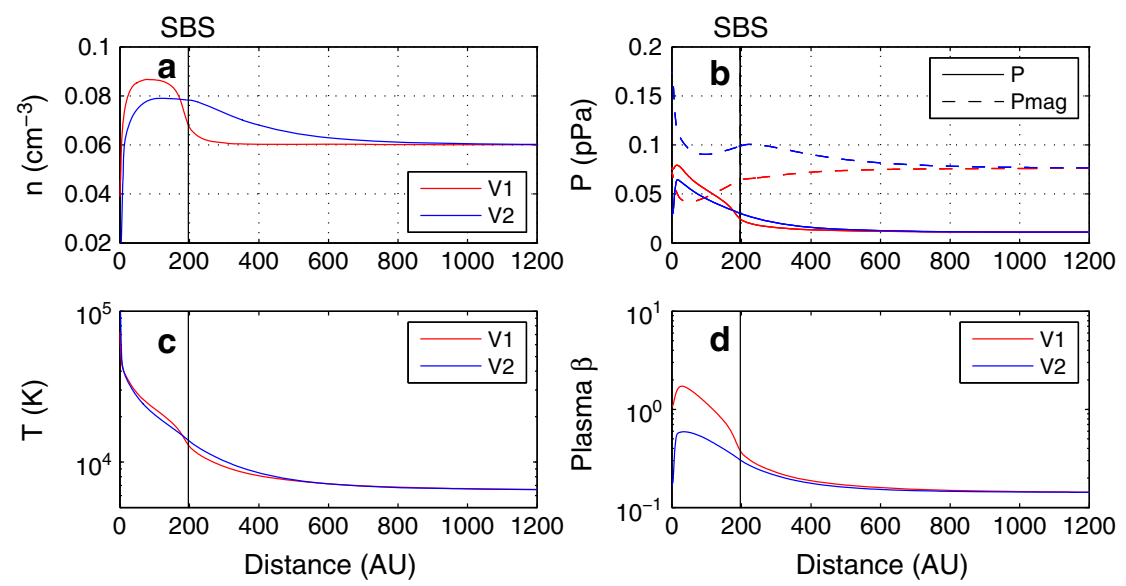

Figure 4. (a) Ion number density, thermal plasma (solid lines), and magnetic (dashed lines) (b) pressures, (c) ion temperature, and (d) plasma $\beta$ along the V1 (red lines) and V2 (blue lines) trajectories beyond the heliopause. Distance is measured from the heliopause (zero point in the figure), which is located at a radial distance of 165 AU and 130 AU from the Sun in the V1 and V2 directions, respectively. The location of the SBS along the V1 trajectory is marked with a vertical black line. Note that there is no SBS along the trajectory of V2.

difference in plasma $\beta$ (Figure $4 \mathrm{~d}$ ). Another fundamental difference is manifested in the magnetic pressure, which shows an increasing trend (due to magnetic pileup) from the pristine LISM toward the heliopause along the V2 trajectory (blue dashed line in Figure $4 \mathrm{~b}$ ) and a decreasing trend across the SBS toward the heliopause along the V1 trajectory (red dashed line in Figure 4b), except for a narrow pileup region close to the heliopause. Such a gradual decrease of magnetic field intensity in the downstream direction is a typical feature of slow shocks.

\section{Discussion and Conclusions}

[22] Our multifluid multi-ion MHD simulation clearly confirmed the theoretically expected quasi-parallel SBS ahead of the heliosphere for the LISM parameters chosen. The simulated SBS is substantially displaced from the axis of the heliosphere (nose direction) because the group velocity of slow magnetosonic waves is quasi-parallel to the ambient magnetic field.

[23] A question to ask is to what extent kinetic effects can damp slow shocks. As the plasma $\beta$ increases, kinetic effects become more and more important, and in the high- $\beta$ limit, the propagation of slow magnetosonic waves is strongly hindered by Landau damping. Hada and Kennel [1985] showed that slow magnetosonic waves can steepen into a shock only if $\beta<1$ and $T_{i} / T_{e}$ is small, where $T_{i}$ and $T_{e}$ are the ion and electron temperatures in the plasma, respectively. However, the situation is different in case of an obstacle, where a slow shock can still exist even in a high- $\beta$ plasma, as pointed out by Karimabadi et al. [1995].

[24] In our model, the plasma $\beta$ in the pristine LISM is as low as $0.14, M_{s}$ must be not more than 2 , and $T_{i} / T_{e}$ is assumed to be around 1 , all of which are favorable for the propagation and steepening of slow magnetosonic waves into a SBS.

[25] V1 is heading more toward the quasi-parallel SBS in our model scenario, whereas V2 would not encounter the SBS because the latter does not exist in the quasiperpendicular region. V1 could encounter a slow, hot, and compressed interstellar plasma population in the outer heliosheath with substantially reduced interstellar magnetic field, while V2 could enter into a less disturbed interstellar wind, where magnetic pressure dominates over plasma pressure.

[26] Since fast magnetosonic waves can propagate upstream of the SBS, the magnetic field and the flow velocity starts to decrease well upstream of the SBS, as demonstrated in Figures $3 \mathrm{~b}$ and $3 \mathrm{~d}$, respectively. This means that the slow bow shock is not the ultimate boundary separating the outer heliosheath from the pristine LISM. There is a fast bow wave ahead of the SBS that starts to decelerate the flow and deflect the magnetic field well upstream of the SBS. This is consistent with the analytical model of Whang [2010], which predicts that the effect of the heliopause would penetrate deep into the interstellar space in case of a subfast interaction with small $\alpha_{B v}$. However, between the heliopause and the SBS, slow magnetosonic waves also play a significant role in further decelerating/diverting the subsonic interstellar flow and further diminishing the magnetic field intensity.

[27] Although the hydrogen wall would be theoretically expected even without any bow shock, we find that the hydrogen wall is bounded by a SBS spatially confined to the quasi-parallel region. Our SBS model predicts a thin and dense hydrogen wall that is strongly asymmetric to the axis of the heliosphere, which remains to be tested with $\mathrm{H}$ Ly $\alpha$ absorption spectra from nearby stars. The asymmetric hydrogen wall is a source of slower and hotter interstellar neutrals, which may help to explain the secondary population of interstellar He recently discovered in the IBEX-Lo data [Bzowski et al., 2012].

[28] Acknowledgments. B.Z. and M.O. would like to thank the support by NFS Career Grant ATM-0747654 and IBEX SWRI subcontract E99019MO. This work was carried out as a part of the IBEX mission, which is part of NASA's Explorer Program. The authors thank ISSI for supporting the heliopause international team.

[29] The Editor thanks two anonymous reviewers for their assistance in evaluating this paper.

\section{References}

Bogovalov, S. V. (1994), On the theory of magnetohydrodynamic winds from a magnetosphere of axisymmetric rotators, Mon. Not. R. Astron. Soc., 270, 721-733. 


\section{ZIEGER ET AL.: SLOW BOW SHOCK}

Bzowski, M., et al. (2012), Neutral interstellar helium parameters based on IBEX-Lo observations and test particle calculations, Astrophys. J. Supp. Ser., 198, 12, doi:10.1088/0067-0049/198/2/12.

Chalov, S. V., D. B. Alexashov, D. McComas, V. V. Izmodenov, Y. G. Malama, and N. Schwadron (2010), Scatter-free pickup ions beyond the heliopause as a model for the Interstellar Boundary Explorer ribbon, Astrophys. J. Lett., 716, L99-L102, doi:10.1088/2041-8205/716/2/L99.

Cox, D. P., and L. Helenius (2003), Flux-tube dynamics and a model for the origin of the Local Fluff, Astrophys. J., 583, 205-228, doi:10.1086/344926.

Florinski, V., N. V. Pogorelov, G. P. Zank, B. E. Wood, and D. P. Cox (2004), On the possibility of a strong magnetic field in the local interstellar medium, Astrophys. J., 604, 700-706, doi:10.1086/382017.

Frisch, P. C., S. Redfield, and J. D. Slavin (2011), The interstellar medium surrounding the Sun, Annu. Rev. Astron. Astrophys., 49, 237-279, doi:10.1146/annurev-astro-081710-102613.

Hada, T., and C. F. Kennel (1985), Nonlinear evolution of slow waves in the solar wind, J. Geophys. Res., 90, 531-535, doi:10.1029/JA090iA01p00531.

Heerikhuisen, J., and N. V. Pogorelov (2011), An estimate of the nearby interstellar magnetic field using neutral atoms, Astrophys. J., 738, 29, doi:10.1088/0004-637X/738/1/29.

Heerikhuisen, J., et al. (2010), Pick-up ions in the outer heliosheath: A possible mechanism for the Interstellar Boundary Explorer ribbon, Astrophys. J. Lett., 708, L126-L130, doi:10.1088/2041-8205/708/2/L126.

Izmodenov, V. V., Y. G. Malama, M. S. Ruderman, S. V. Chalov, D. B. Alexashov, O. A. Katushkina, and E. A. Provornikova (2009), Kineticgasdynamic modeling of the heliospheric interface, Space Sci. Rev., 146 329-351, doi:10.1007/s11214-009-9528-3.

Karimabadi, H., D. Krauss-Varban, and N. Omidi (1995), Characteristic speeds in high $\beta$ isotropic/anisotropic plasmas, Phys. Plasma, 2, 4177-4184, doi:10.1063/1.871043.
Kogan, M. (1959), Shock waves in magnetohydrodynamics, J. Appl. Math Mech., 23, 784-792, doi:10.1016/0021-8928(59)90169-8.

McComas, D. J., et al. (2009), Global observations of the interstellar interaction from the Interstellar Boundary Explorer (IBEX), Science, 326 , 959-962, doi:10.1126/science.1180906.

McComas, D. J., et al. (2012), The heliosphere's interstellar interaction: No bow shock, Science, 336, 1291-1296, doi:10.1126/science.1221054.

Opher, M., F. A. Bibi, G. Toth, J. D. Richardson, V. V. Izmodenov, and T. I. Gombosi (2009), A strong, highly-tilted interstellar magnetic field near the Solar System, Nature, 462, 1036-1038, doi:10.1038/nature08567.

Pogorelov, N. V., G. P. Zank, and T. Ogino (2006), Three-dimensional features of the outer heliosphere due to coupling between the interstellar and interplanetary magnetic fields. II. The presence of neutral hydrogen atoms, Astrophys. J., 644, 1299-1316, doi:10.1086/503703.

Prested, C., M. Opher, and G. Tóth (2012), Multi-ion, multi-fluid 3-D magnetohydrodynamic simulation of the outer heliosphere, eprint arXiv: 1211.1908 .

Schwadron, N. A., and D. J. McComas (2013), Spatial retention of ions producing the IBEX ribbon, Astrophys. J., 764, 92, doi:10.1088/0004$637 \mathrm{X} / 764 / 1 / 92$

Schwadron, N. A., et al. (2009), Comparison of Interstellar Boundary Explorer observations with 3D global heliospheric models, Science, 326 , 966-968, doi:10.1126/science.1180986.

Tóth, G., et al. (2012), Adaptive numerical algorithms in space weather modeling, J. Comput. Phys., 231(3), 870-903, doi:10.1016/ j.jcp.2011.02.006

Whang, Y. C. (2010), Interstellar magnetic field surrounding the heliopause, Astrophys. J., 710, 936-940, doi:10.1088/0004-637X/710/2/936.

Witte, M. (2004), Kinetic parameters of interstellar neutral helium. Review of results obtained during one solar cycle with the Ulysses/GASinstrument, Astron. Astrophys., 426, 835-844, doi:10.1051/00046361:20035956. 